\title{
La construcción epistemológica en Ingeniería Civil: Visión de la Universidad de Costa Rica
}

The epistemological construction in Civil Engineering: the vision of the University of Costa Rica

\author{
Volumen 19, Número 1 \\ Enero-Abril \\ pp. $1-30$
}

Este número se publica el 1 de enero de 2019

DOI: 10.15517/aie.v19i1.35328

Nidia Cruz Zúñiga

Erick Centeno Mora

Revista indizada en REDALYC, $\underline{\text { SCIELO }}$

Revista distribuida en las bases de datos:

LATINDEX, DOAJ, REDIB, IRESIE, CLASE, DIALNET, SHERPA/ROMEO, QUALIS-CAPES, MIAR

Revista registrada en los directorios:

ULRICH'S, REDIE, RINACE, OEI, MAESTROTECA, PREAL, $\underline{\text { CLACSO }}$ 


\title{
La construcción epistemológica en Ingeniería Civil: Visión de la Universidad de Costa Rica
}

The epistemological construction in Civil Engineering: the vision of the University of Costa Rica

\author{
Nidia Cruz Zúñiga ${ }^{1}$ \\ Erick Centeno Mora²
}

\begin{abstract}
Resumen: Definir el ámbito de estudio de una disciplina es un requisito indispensable para su desarrollo académico y pedagógico. Para la Ingeniería Civil, en Costa Rica, no existe registro de que este ejercicio se haya realizado anteriormente. En el presente artículo se sintetiza el proceso de construcción del marco epistemológico de la Ingeniería Civil en Costa Rica como parte del proceso de actualización del perfil de egreso del estudiantado de Licenciatura en Ingeniería Civil de la Universidad de Costa Rica. Se utilizó un abordaje mixto, cualitativo y cuantitativo, junto con la observación participativa de una comisión de docentes conformada por 14 personas y creada dentro de la Escuela de Ingeniería Civil, con el fin de atender específicamente este reto. Se realizaron consultas por medio de entrevistas, talleres y encuestas a poblaciones clave. Las muestras comprenden personas docentes (74), personas investigadoras (10), personas profesionales destacadas (10), estudiantes (278), personas graduadas (1506) y empleadoras (306). Las respuestas de las distintas poblaciones de estudio fueron contrastadas entre sí y complementadas con referentes internacionales. Los principales resultados de la investigación llegaron a la definición adaptada al contexto local de conceptos clave: ingeniería, ingeniería civil y sus especialidades. También fueron identificadas transformaciones históricas de la disciplina en Costa Rica, las cuales han repercutido significativamente en cómo se enseña y se aprende en la carrera. Finalmente, se logró identificar algunos cambios que complementan una visión tradicional de la disciplina con la revolución tecnológica actual.
\end{abstract}

Palabras clave: epistemología, ingeniería civil, perfil de egreso, pedagogía en ingeniería

\begin{abstract}
Defining the scope of a discipline is indispensable for its academic and pedagogical development. For the civil engineering in Costa Rica, there is no precedent for this task in the past. This article aims to synthetize the process of construction of an epistemological frame for Civil Engineering in Costa Rica, as part of an updating process for the graduate profile for civil engineer students at the University of Costa Rica. A qualitative and quantitative approach; along with a participative observation within a teacher commission integrated by 14 people that was created in the Civil Engineer School specifically for that purpose. Queries through interviews, workshops and inquiries were applied to stakeholders. The samples were composed of teachers (74), researchers (10), featured professionals (10), students (278), graduates (1506) and employers (306). The answers of each population were compared and completed with international references. The main results of this research are a definition, adapted to the local context, of key concepts for establishing an epistemology for civil engineering: engineering, civil engineering and its specialties. In addition, historical transformations on how the formation is taught and how it is learnt were identified. Finally, some changes derived from the current technological revolution that would complement the traditional vision of the discipline were identified.
\end{abstract}

Key Words: epistemology, civil engineering, graduate profile, engineering pedagogy

\footnotetext{
1 Profesora de la Universidad de Costa Rica en la Escuela Ingeniería Civil. Licenciada en Ingeniería Civil, Master en Medio Ambiente con mención en Ingeniería del tratamiento de residuos, Master en Evaluación de Programas y Proyectos. Dirección electrónica: ncruzzz@gmail.com

2 Profesor de la Universidad de Costa Rica en la Escuela Ingeniería Civil. Licenciado en Ingeniería Civil, Master en Gestión y Tratamiento del agua, el suelo y los desechos. Dirección electrónica: ecenteno86@gmail.com
}

Artículo recibido: 11 de abril, 2018

Enviado a corrección: 11 de setiembre, 2018

Aprobado: 29 de octubre, 2018 


\section{Introducción}

El presente artículo expone parte de los resultados obtenidos en una investigación más amplia realizada en la Escuela de Ingeniería Civil de la Universidad de Costa Rica. Dicha investigación se efectuó con miras a la actualización del perfil de egreso de la Licenciatura en Ingeniería Civil y del plan de estudios como tal. Abarcó la capacitación docente en temas relacionados con la enseñanza superior y con los principios teóricos de la construcción de conocimiento que se deben propiciar en la universidad. La investigación se realizó entre junio de 2016 y agosto de 2018, contempló una metodología que concatenó diferentes herramientas investigativas para recopilar información relevante desde las diferentes aristas: la academia, el mercado laboral, la realidad de la sociedad costarricense, los referentes internacionales y los actores clave implicados en este campo disciplinar (para este último punto se construyó un mapeo de actores, en el que se priorizó la consulta directa de quienes la comisión docente, nombrada para el proceso, determinó como más importantes).

Al iniciar el proceso para conocer el objeto de estudio de la carrera se evidenció la poca información escrita, científica y publicada, que existe al respecto, por lo que se consideró relevante la realización de un esfuerzo adicional para plasmar los resultados obtenidos en la investigación y sentar una base concreta acerca de la epistemología de la disciplina en Costa Rica. Este esfuerzo contribuyó a la profundización de la reflexión, en especial, enfocada a los procesos de investigación que se llevan a cabo en el país.

En este artículo, primero se presentan los postulados teóricos que permiten definir una epistemología en la ingeniería, necesarios para la revisión curricular de la carrera de Licenciatura en Ingeniería Civil de la Universidad de Costa Rica. Posteriormente, se explica la metodología abordada, la cual contempló herramientas cuantitativas y cualitativas para la obtención de los datos necesarios para el análisis y construcción de conocimiento en el área. Este abordaje mixto enriqueció la pesquisa, y propició un abordaje más integral de la realidad del desarrollo de la investigación en la disciplina. Esta estrategia multimétodo fue seleccionada por las personas autoras con el fin de tener una combinación de bondades y que se propiciara la triangulación metodológica. Tal y como establece Arias (2000, p. 04) la triangulación es el uso de múltiples métodos en el estudio de un mismo objeto, y conviene concebirla contemplando variedad de datos, personas investigadoras y teorías.

Seguidamente, se presentan los resultados de la investigación. Se hace énfasis en las definiciones propias de la disciplina y sus áreas, resultado de los procesos de consulta y de 
la construcción colectiva. Adicionalmente, se exponen los cambios en las áreas y temáticas que se investigan en la actualidad, y los retos que la disciplina requiere trabajar a futuro respecto de la realidad social y cultural en la que están inmersas la carrera y la Universidad.

\section{Referentes teóricos}

Para hablar de epistemología se requiere de un abordaje profundo y riguroso de la disciplina, el cual implica empezar a cuestionar todo lo que se conoce y se hace en el campo de acción de la carrera para abrir las puertas a nuevos retos y cambios de la disciplina en un contexto que siempre es cambiante. Tal como comenta Vasilachis (2009, p.03):

La epistemología se interroga, entre otros, acerca de: a) cómo la realidad puede ser conocida, b) la relación entre quién conoce y aquello que es conocido, c) las características, los fundamentos, los presupuestos que orientan el proceso de conocimiento y la obtención de los resultados, y d) la posibilidad de que ese proceso pueda ser compartido y reiterado por otros a fin de evaluar la calidad de la investigación y la confiabilidad de esos resultados.

Por ello, el punto de partida del proceso de investigación, asociado a la reforma curricular de la carrera, arrancó con un análisis profundo de lo que existe en cuanto a definiciones de la disciplina, tanto a nivel nacional como internacional, para analizarlo de acuerdo con la realidad de la carrera desde la Universidad de Costa Rica y de la Escuela de Ingeniería Civil. Además, se aprovechó para revisar las reflexiones que otras personas autoras han hecho al respecto. Entre quienes ha abordado ampliamente el tema del estatus epistemológico de la Ingeniería se encuentran artículos escritos por personas educadoras, filósofas y algunas ingenieras (Bulleit, 2012; Coronado, 2002; Covarrubias, 1998; Dias, 2013; Figueiredo, 2008; Galland, 2011; Herrera, 2016; Koen, 2003; Poser, 1998). En el caso particular de Costa Rica, existen muy pocos registros formales de estudios en la materia, más bien, se han centrado en documentos propios de la Universidad de Costa Rica donde se analiza el tema para la justificación curricular. Uno de los autores que más rigurosamente ha escrito sobre epistemología en ingeniería en Costa Rica ha sido Herrera (2016), quien ha presentado artículos donde cuenta la historia del desarrollo de la profesión en el país; sin embargo, este autor no es propio de la ingeniería civil, sino que aborda el tema de forma general para la ingeniería, y hace énfasis en las diferencias entre tecnología e ingeniería. 
Hablar de epistemología tiene una trascendencia importante para la actualización curricular en una carrera, pues contribuye a que las personas encargadas de ella aclaren cuál es su objeto de estudio y cómo interactúan los seres humanos a través de ella. La reflexión sobre la reforma curricular del Programa de Formación en Ingeniería Civil inicia reflexionando sobre el objeto de estudio al que responde y cómo este ha evolucionado en el tiempo. La evolución de la disciplina de ingeniería civil nace, principalmente, de la investigación que se realiza en las universidades, donde más se invierten recursos con este fin; no obstante, en el caso particular de la ingeniería civil, los cambios también se forjan desde las empresas públicas y privadas.

En Costa Rica, el Colegio Federado de Ingenieros y de Arquitectos, encargado de velar por el adecuado desarrollo de la práctica profesional, también tiene participación en la creación de conocimiento en ingenieria. Como indica Vasilachis (1992, p. 03), la reflexión epistemológica está presente en la actividad cotidiana de investigación —aunque la persona que investiga la lleve a cabo sin darle ese nombre - al plantearse interrogantes acerca de las características del objeto o de los fenómenos que analiza. Es por ello que uno de los puntos de partida de este proceso de investigación fue indagar qué novedades están surgiendo en los diferentes ámbitos laborares de la disciplina. Como se discutirá en la metodología, esto se realizó mediante un abordaje cualitativo, utilizando como instrumento la entrevista a profundidad para personas investigadoras y con un abordaje cuantitativo mediante encuestas a personas empleadoras.

Al plantearse la tarea de un estudio epistemológico hay que tener claro que "la realidad de las diferentes prácticas científicas reside en su distinción, cada una tiene su propio objeto, su teoría, sus métodos y su desarrollo desigual” (Lecourt, 1985, citado por Vasilachis, 1992, p.14). Esta realidad también enmarca el desarrollo de la Ingeniería Civil en la Universidad de Costa Rica, pues dentro de esa misma disciplina coexisten diferentes áreas, y cada una de ellas tiene su propio objeto de estudio. Aunque estos objetivos se entrelazan, quienes se dedican a la ciencia y la investigación por lo general tratan de visualizarlos individualmente. Los paradigmas que rigen la investigación en cada área pueden ser distintos o similares, pero, en general, surgen de los aportes teóricometodológicos más relevantes en el campo y de las necesidades que la sociedad va planteando como retos a la disciplina. 


\subsection{Ingeniería, ciencias básicas, ciencias de la ingeniería y tecnologías}

El primer punto de discusión en el análisis epistemológico de este proceso radica en distinguir a cuál ámbito del conocimiento pertenece esta disciplina. Existe un consenso entre las diferentes personas autoras consultadas respecto a que la ingeniería es un campo del conocimiento de difícil delimitación. La ingeniería reúne otras áreas del conocimiento, tales como ciencias básicas, ciencias aplicadas, ciencias de la ingeniería y ciencias de la tecnología aplicada, y las utiliza para aplicarlas en la resolución de problemas. En este punto, se hace necesario un razonamiento más amplio sobre esta cuestión para contribuir a clarificar el alcance de la ingeniería.

Para empezar, Dias (2013, p.23) explica que "una clara distinción entre el conocimiento puramente científico y el ingenieril es que el primero es utilizado para explicaciones causales que permiten una mejor comprensión del mundo, generando así información etiológica, mientras que el otro genera información teleológica". Cuando una persona ingeniera piensa en la definición de ingeniería, una de las primeras ideas que surge es la resolución práctica de problemas, para ello aplica una serie de conocimientos de ciencias básicas y aplicadas, ayudada por las herramientas que el desarrollo tecnológico pone a su disposición, lo que le permite tomar decisiones sobre una solución razonable al problema. Se hace hincapié en lo factible y razonable porque, a diferencia de otras disciplinas, la persona profesional en ingeniería suele partir de la premisa de que la solución que busca no es perfecta ni única, pero es realizable con los recursos con los que cuenta, y espera que el desempeño de dicha solución sea aceptable dentro de los parámetros de seguridad y durabilidad que seleccionó para el caso.

Galland (2011, p.05) señala que "el investigador de las ciencias básicas o naturales trabaja en los temas que le interesan por motivos puramente cognoscitivos, mientras que el investigador aplicado y el ingeniero estudian temas de posible interés social”, donde se desea encontrar una solución a problemas contextualizados y bien definidos. Es decir, la labor ingenieril responde a una realidad, se debe a una necesidad de la sociedad donde actúa, por ende, la ingeniería debe reflejar un fuerte componente social que a veces no está tan visibilizado en los proyectos puntuales que resuelve. Para Poser (1998, p.83), el o la profesional en ingeniería no desea necesariamente un conocimiento más profundo de una situación, sino, más bien, una "mejor" solución para el problema que se le plantea. Muchas veces a la persona ingeniera no le interesa ir a lo profundo en términos etiológicos, sino 
simplificar el problema lo mejor posible para controlar las variables de análisis y así, modelar mejor lo que puede ocurrir con la solución que se está planteando.

De manera análoga Prince y Llach (2006, p. 04) señalan que las ciencias básicas y las ingenierías tienen diferentes métodos y persiguen objetivos distintos. La ciencia "pura" busca una verdad universal, mientras que la ingeniería persigue un fin utilitario. Si quien es profesional en ingeniería considera que su trabajo no es útil, no invierte tiempo en ello, se le entrena para ser eficiente en el uso de los recursos, entre ellos el tiempo. Para quien investiga en ingeniería, todo su esfuerzo tiene como razón de ser asociada a resolver una determinada necesidad o crear una nueva solución al problema que está analizando; enfatiza en mejorar sin que ello lo lleve a la perfección. Para la persona especialista en ingeniería no sería necesario estar aplicando una verdad universal o absoluta, ejemplo de ello es que utiliza teorías de la mecánica newtoniana para resolver gran cantidad de los problemas a los que se enfrenta, a sabiendas de que existen otras teorías más universales para comprender el universo de la física.

Esta diferencia fundamental entre la ingeniería y las ciencias básicas o naturales determina también diferencias importantes en las metodologías y modos de proceder en las diferentes áreas, así como la manera en la que se aprende y se enseña cada una de ellas. Al comparar la ingeniería con las ciencias de la ingeniería o ciencias aplicadas, Bulleit (2012, p. 1145) explica que la primera hace uso importante de las segundas, el motivo, según ese autor, es el contexto de contingencia y de incertidumbre en que las personas que trabajan en ingeniería deben tomar decisiones. Quien ejerce la ingeniería deben tener la claridad de que los modelos simplificados de las ciencias aplicadas o ciencias de la ingeniería que emplea pueden omitir información que se debe considerar durante los proyectos reales. Ciertamente, esas ciencias son indispensables para el ejercicio de la ingeniería, pues permiten crear un modelo matemático o físico que posibilita encontrar una solución medible. No obstante, son insuficientes para explicar el modo de proceder de la ingeniería.

Cuando se considera la diferencia de la ingeniería con respecto a la tecnología aplicada, Poser (1998, p. 91) señala que "la capacidad de interpretación de una situación particular que requiere un ingeniero trasciende lo meramente tecnológico, pues se requiere juzgar esa situación en un contexto de valores éticos anclados en un contexto social específico". Adicionalmente, Bulleit (2012 p. 1149) explica que, aunque el tecnólogo y el ingeniero compartan algunos elementos en relación con el "saber cómo" (know how), que 
aplican por medio del diseño de artefactos que satisfagan las necesidades de una situación específica, existe una diferencia en el nivel de profundización de los métodos heurísticos de la ingeniera y la tecnología.

Para la presente investigación, la diferencia entre la ingeniería y la tecnología radica ciertamente en la contextualización de los problemas a resolver, y en la particularidad que esto le puede dar a cada situación. Esto ocurre, como argumentó Bulleit, por la aplicación ética de los conocimientos ingenieriles a la resolución de problemas. También se considera que hay diferencia en la aplicación de los métodos y las teorías, dicha diferencia es la que se explica a continuación: en el caso del o la profesional en ingeniería, los métodos van desde concepciones "mundanas", como por ejemplo, diseño por medio de tiempo de retención hidráulica, o "reglas de dedo", rule of thumb, tales como reglas para sumar decibeles de forma directa, hasta modelos más abstractos, como los conceptos de flujo en canales abiertos y técnicas avanzadas de análisis estructural que requieren una mejor comprensión de la naturaleza. En ese sentido, a diferencia de la persona profesional en tecnología aplicada, la persona profesional en ingeniería requiere una sólida base conceptual de las ciencias de la ingeniería, que le permitan tener conceptos claros a la hora de hacer uso de los métodos heurísticos que pone en práctica.

\subsection{Concepción teórica y metodológica de la ingeniería}

Como un área específica del conocimiento humano, la ingeniería tiene sus propios métodos para alcanzar sus objetivos, los ha desarrollado a lo largo de muchos años y, en gran medida, mediante prueba y error. Algunos de estos métodos son antiguos, pero se siguen considerando válidos, siempre que no se encuentre una mejor forma de aproximar la modelación a la realidad. Por ejemplo, en la ingeniería es común la utilización de monogramas que han sido desarrollados de forma empírica o con experimentación para un contexto determinado; sin embargo, en ausencia de otras herramientas, se consideran válidos para la resolución de problemas, siempre que se expliquen las limitaciones del caso.

Bulleit (2012, p. 1144) explica que, "en el afán de resolver los problemas de la sociedad, los ingenieros diseñan artefactos grandes o pequeños". En el caso de la ingeniería civil esos artefactos son obras como puentes, edificios, carreteras, plantas de tratamiento de aguas residuales, entre otros. 
Según este autor, desarrollar esa habilidad para diseñar debería ser precisamente el fin último de la educación en ingeniería. En este sentido, el proceso de diseño en ingeniería debería retomar el uso de las ciencias de la ingeniería, un razonamiento heurístico, una habilidad de visualizar algo que todavía no existe y un "saber cómo" crear, pasando algunas veces por un proceso de "prueba y error" (Bulleit, 2012, p.1145). A lo anterior se añade el análisis de un contexto social específico, indisoluble de la factibilidad de desarrollar las soluciones con los recursos y las limitaciones presentes.

Por la importancia del diseño que se planteó anteriormente, algunos entes de acreditación de carreras de ingeniería, como el Comité de Acreditación en Ingeniería de Canadá (CEAB, por sus siglas en inglés), por ejemplo, hacen énfasis en la cantidad de horas de diseño en los programas de formación en ingeniería. Como ocurre con el diseño en ingeniería, esos entes acreditadores también promueven que la formación considere otros atributos o cualidades que contribuyen al desarrollo del futuro profesional en ingeniería, tales como la habilidad de investigación, las habilidades de expresión oral y escrita, el profesionalismo, la ética, entre otros. De este modo, el diseño sería una cualidad medular, aunque insuficiente por sí sola, para la educación y el ejercicio de la ingeniería en la actualidad.

Retomando el uso de la heurística en la ingeniería, personas que han escrito del tema como Dias (2013, p. 23), Figuereido (2008, p. 1), Poser (1998, p. 86) y Prince y Llach (2006, p. 4) concuerdan en que el diseño es precisamente un elemento distintivo de esta disciplina. Bulleit (2012, p. 1146) define la heurística como "cualquier cosa que provea una idea o ayuda plausible de la dirección a tomar en la solución de un problema, pero que puede ser injustificable e inclusive puede fallar". Esto no dista de lo sugerido por la raíz misma de la palabra "heurística" (y del ¡Eureka! atribuido a Arquímides), heurískein, esto es, encontrar una solución a partir de la diversidad de hallazgos que se van obteniendo en el camino. Cuando la persona ingeniera está ejerciendo la profesión es precisamente eso lo que hace en muchos de los casos: probar posibles modelos que aproximen las soluciones hasta encontrar la que mejor se adapte a las circunstancias particulares, a sabiendas de que, en el camino, sus diseños o ideas pueden cambiar.

Una persona profesional en ingeniería debe tomar decisiones con base en conocimientos muchas veces limitados y ante condiciones de alta incertidumbre, para lo cual emplea métodos heurísticos. Esta manera de proceder de la ingeniería es bastante distinta 
del método científico de razonamiento por deducción, pues, en el primer caso, se suele usar un procedimiento de razonamiento inductivo (Bulleit, 2012, p. 1149), pasando de condiciones muy específicas (un problema contextualizado) a un modelo general que permita encontrar una solución al problema concreto. Una vez encontrada dicha solución, debe regresarse al contexto y situarla en las condiciones sociales, ambientales y hasta políticas que se tienen para valorar si es factible su implementación.

En las palabras de Koen (2003, p. 94), el método de resolución de problemas en ingeniería es el uso de la heurística para causar un cambio positivo en una situación pobremente entendida, y en concordancia con los recursos disponibles. Para este fin, y con base en el método heurístico, en ingeniería se suelen emplear tanto teorías científicas como tecnológicas. Las teorías científicas corresponden a las formuladas desde las ciencias básicas y tienen por objetivo facilitar un mejor entendimiento del problema y el medio circundante. Las teorías tecnológicas, por el contrario, son teorías que tienen un fin más práctico que cognitivo. Se dividen en teorías sustantivas y operativas (Galland, 2011, p. 06), y también son usadas por la ingeniería, como se explica a continuación.

Las teorías tecnológicas sustantivas de las que habla Galland (2011, p. 06) suministran conocimiento sobre el objeto de una acción y se derivan de teorías científicas. Por ejemplo, una teoría científica podría ser "el agua hierve a $100^{\circ} \mathrm{C}$ ", mientras que la teoría sustantiva, de porte más práctico, podría enunciarse como "para hervir agua (el objetivo práctico), caliéntela hasta llegar a $100^{\circ} \mathrm{C}$. Un ejemplo aplicado a la Ingeniería Civil, entre estos dos tipos de teorías, se da en la mecánica de suelos como teoría científica, y la geotecnia como teoría tecnológica, por cuanto esta última pretende usar los conceptos de la primera para resolver casos concretos.

Las teorías tecnológicas operativas estudian las relaciones complejas ser humanomáquina en situaciones más o menos reales (Galland, 2011, p. 7). Para ilustrar, en el estudio de los medios de transporte se utilizan teorías tecnológicas operativas que pretenden estudiar el comportamiento del sistema y la relación entre las distintas variables (más que estudiar los vehículos per se), con el fin de generar conocimiento para la toma de decisiones (ejemplo de un objetivo práctico: optimizar un sistema de transportes de una ciudad).

A partir de esas teorías tecnológicas se generan reglas tecnológicas también (o enunciados nomopragmáticos) (Galland, 2011, p. 8), que serían "recetas prácticas" sobre cómo actuar, en determinados casos, para alcanzar el fin deseado. Muchas veces, estas 
"recetas" serán utilizadas por otras personas ingenieras sin cuestionar mucho su trasfondo, buscando el beneficio de una solución rápida y asumiendo la incertidumbre asociada, hasta que se cuente con una "receta mejor". El diseño heurístico en la ingeniería se guía muchas veces por esas reglas tecnológicas, establecidas por la buena praxis y por los diferentes códigos de diseño que ya alguien más estudió a profundidad. Ciertamente, existen profesionales y académicos en ingeniería que, con criterios científicos, buscan mejorar dichas reglas o recetas para ponerlas a disposición de las demás personas colegas.

En síntesis, toda la ingeniería emplea métodos heurísticos, fijados en las teorías, y reglas tecnológicas con el fin de diseñar artefactos (en el caso de la ingeniería civil estos artefactos son las infraestructuras o servicios) que permitan resolver problemas específicos de la sociedad. Ahora bien, existen muchas ramas de la ingeniería civil, la diferencia entre ellas radica en el tipo de problema de la sociedad que cada una aborda. Esto se abordará más adelante en detalle.

\section{Metodología}

Como se indicó, la investigación que dio pie a este artículo partió de la reforma curricular de la carrera de Ingeniería Civil de la Universidad de Costa Rica, y aquí se presentan los resultados del componente epistemológico. A continuación, se explica la metodología utilizada para la totalidad de la investigación. La metodología fue diseñada en concordancia con los lineamientos del Centro de Evaluación Académica (CEA), concernientes a los procesos de diseño y revisión curricular para todas las carreras de la Universidad de Costa Rica. Dicha revisión curricular favorece la reflexión colectiva acerca de los diferentes marcos que deben ser analizados para definir el perfil de egreso de una carrera; es decir, es un análisis curricular cuyo principal objetivo es actualizar el perfil de acuerdo con el estudiantado como actor central.

La parte de la investigación que le atañe a este artículo se circunscribe al estudio de la epistemología de la disciplina. Se partió de un análisis situado que comprende el desarrollo de la disciplina como un proceso político-educativo en el que participan diferentes actores clave (docentes, estudiantes, personas graduadas, personas empleadoras y sociedad civil) en el marco institucional de la Universidad de Costa Rica.

Se plantearon una serie de criterios derivados de las dimensiones epistemológica, socio-profesional y pedagógica, propias del currículo universitario y, a partir de ello, se re- 
contextualizó el objeto de estudio. La investigación reconoció los dos grandes ámbitos del currículo: el estructural-formal, que incluye todas las disposiciones oficiales emanadas, tanto de la Institución en sentido amplio como del cuerpo docente organizado; y el procesalpráctico, que implica todo aquello que sucede a partir de lo establecido en la documentación oficial y que, finalmente, solicita el reconocimiento de todas aquellas prácticas cotidianas en las aulas, y que responden a las particularidades de la disciplina. La concatenación de estos dos ámbitos permitió un mejor abordaje de la realidad para conocer ¿qué es lo que se debe estudiar y por qué?

En cuanto a la operacionalización para la recopilación de información se diseñaron una serie de instrumentos de consulta. Estos instrumentos, cuestionarios para las encuestas y guías de entrevistas, fueron elaborados por las personas autoras de este artículo y validados por el grupo de docentes que trabajó en la reforma curricular. Se realizó un mapeo de actores clave ("stakeholders") (listado de personas implicadas en el tema de investigación y las interrelaciones entre ellas), con base en ello se decidió el tipo de instrumento de consulta que se utilizaría con cada caso. Tapella (2007, p.3) habla del mapeo de actores clave y establece que es una metodología que supone el uso de esquemas para representar la realidad social del proyecto, para comprenderla en su extensión más compleja y establecer estrategias de cambio para la realidad así comprendida.

Según esta definición se construyó el mapeo de actores clave con el fin de entender la dinámica social en la que la carrera está inmersa. Posteriormente, para el análisis, se consideraron los diferentes aportes de las poblaciones de acuerdo con su relación con la carrera y se valoró el peso de sus intervenciones según el grado de experiencia que la persona informante tenga respecto al desarrollo de la disciplina. En este sentido, se consideraron los aportes de personas expertas e investigadoras como muy valiosos, al punto que la información que ellas aportaron se manejó de forma muy puntual. Los resultados de las entrevistas a profundidad fueron validados posteriormente en un taller participativo con otra población clave (personas docentes activas de la Escuela) mediante el uso de dinámicas grupales por área de especialización, para así triangular la información y obtener mayor claridad de las dimensiones asociadas al objeto de estudio.

Para poblaciones grandes se decidió trabajar con encuestas en línea: estudiantes, docentes, personas graduadas y empleadoras (más adelante se detallan los aspectos de estas consultas). Para poblaciones pequeñas se optó por un instrumento cualitativo que 
fuera flexible y a la vez permitiera indagar con mucha más profundidad la opinión de la persona. En este caso, se utilizó la técnica de las entrevistas a profundidad. Este último tipo de instrumentos se utilizó con personas profesionales expertas e investigadoras. Asimismo, se analizó la realidad de cada población y el fin que se perseguía al consultarla, y con base en ello se planteó un abordaje mixto que busca rescatar los mejores aportes de cada método, pues, tal como indica Vasilachis (1992, p. 57):

Los métodos cuantitativos se enmarcan en una concepción positivista, que aplica controles rígidos a situaciones artificiales, y en cuya aplicación el investigador intenta operar manteniendo cierta distancia y neutralidad. En los métodos cualitativos se actúa sobre contextos reales y el observador procura acceder a las estructuras de significados propias de esos contextos mediante su participación en los mismos.

Para la participación de las personas entrevistadas en este proceso se contó con el consentimiento informado respecto a sus aportes, cómo estos iban a ser utilizados y asociados a sus nombres. Para el caso de las consultas cuantitativas, se indicó al inicio del instrumento que los aportes eran anónimos y que se iban a manejar en estadísticas globales, no se solicitó el nombre de la persona encuestada, sino solo algunas características particulares para clasificar la información obtenida. Finalmente, en las mesas de discusión y talleres sí se le indicó a cada participante que su aporte iba a ser asociado a su nombre, área de especialización e incluso a su prestigio como profesional.

Cabe destacar que las investigaciones tradicionales en ingeniería suelen utilizar más los métodos cuantitativos, pero para la presente investigación fue clave el abordaje desde la arista cualitativa, pues la complejidad de los problemas que se abordan y del objeto de estudio en sí, hace que sea necesario el uso de métodos más robustos y flexibles a la vez. Parte de las premisas de análisis definidas contempla que el mundo requiere personas ingenieras con conciencia social. Velásquez y D' Armas (2015, p .02) lo plantean como una ingeniería que busca atender la responsabilidad implícita de conservar un ambiente sano para las generaciones futuras, en el contexto del desarrollo sostenible, lo que plantea el reto de lograr ver la ingeniería civil como una ingeniería más social, que responde a necesidades reales y a un público muy humano, el cual está enfrentando problemas complejos que requieren de abordajes multidisciplinares para ser solucionados. 
La recopilación de información primaria vía consulta a poblaciones claves se realizó entre diciembre de 2016 y marzo de 2017 (ver detalles del número de personas por población en la Tabla 1). Las poblaciones que se consideraron para ser parte del estudio eran variadas, en algunos casos se sabía el tamaño completo de la población y en otros se desconocía, se trabajó con los datos que se lograron recopilar. Para cada una de dichas poblaciones se contemplaron criterios de selección pertinentes, los cuales detallan a continuación:

- Estudiantes: Se conoce la totalidad de la población, pues se consultó a la población estudiantil activa que se encontraran cursando 7, 8, 9 o 10 ciclo lectivo en el segundo semestre de 2016 (según su avance en el plan de estudios), además de aquellas personas egresadas que estaban únicamente con su trabajo final de graduación. Se consideró que, a partir de estos niveles en la carrera, los y las estudiantes ya tienen suficiente conocimiento de su carrera para emitir un criterio al respecto. Se tuvo el cuidado de asociar el ciclo lectivo de la persona encuestada a sus respuestas a la hora de realizar el análisis y valorar el peso de estas. En este caso, se realizó un sondeo a toda la población disponible con el objetivo de que respondiera la mayor cantidad de individuos posible. A esta población se le consultó aspectos didácticos y pedagógicos, en especial, relacionados con la calidad de los cursos y la adecuación de cada uno de ellos dentro del currículum de la carrera. Aunado a ello, se les consultó sobre los ejes temáticos que aborda la carrera, la forma cómo ellos y ellas logran construir su aprendizaje, los atributos (habilidades) que se les va desarrollando, entre otros. El instrumento de consulta responde a un enfoque cuantitativo mediante encuesta cerrada.

- Docentes: se contempló la totalidad de las personas docentes nombradas para el segundo semestre del año 2016, tanto personas interinas como propietarias. Se decidió que, en este caso, la muestra debería ser prácticamente la totalidad de la población, por lo que se trabajó en ello y se logró un muy alto porcentaje de respuesta (84\% como se verá en la Tabla 1). A esta población se le consultaron aspectos más de fondo del saber, saber ser y saber hacer de la profesión desde el punto de vista académico. Se especificaron consultas respecto a contenidos y objetivos de los cursos, métodos de enseñanza - aprendizaje, estrategias didácticas utilizadas, entre 
otros. El abordaje con esta población también corresponde a un enfoque cuantitativo mediante encuesta cerrada.

- Personas graduadas: se contemplaron todas las cohortes de estudiantes que se han graduado con el actual plan de estudios. Para ello, se solicitaron las listas del estudiantado graduado desde el año 2000 al año 2016 a la Oficina de Registro. Luego se depuró la base de datos con aquellos correos que eran todavía válidos, y se hizo un seguimiento telefónico para invitar a responder la encuesta o corregir datos de cada contacto. En este caso, la muestra que se requería se definió en al menos el 10\% de la población, dada la cantidad de individuos que la conformaban (refiérase a la Tabla 1 para el detalle del número de individuos en la muestra y la población). A esta población se le consultó sobre las habilidades y conocimientos adquiridos y cómo estos les han sido útiles para el desempeño profesional, aunado a cómo su proceso de enseñanza aprendizaje les ayudó a adaptarse al contexto y a las demandas del mercado. La técnica de recopilación de información en esta población correspondió a un instrumento cuantitativo, también de entrevista cerrada.

- Personas empleadoras: se levantó una lista con base en el criterio y conocimiento de cada docente que participó en el proceso de revisión curricular, lo que implicó contemplar las diferentes áreas disciplinares, la representatividad por sectores productivos y el tener un contacto directo con la empresa o institución. Una vez montada la base de datos, se procedió, por medio de llamadas telefónicas, a invitarlos a participar del proceso y a corroborar los datos del contacto. A esta población fue a la que más seguimiento se le dio, esto para alcanzar el nivel de respuesta obtenido. La muestra que se planteó también consideró alcanzar al menos el $10 \%$ de la representación seleccionada inicialmente por las referencias de las personas docentes que participaron en la reforma curricular (ver número de participantes en la Tabla 1). La población total de personas empleadoras es un número desconocido, no se tienen registros en la Escuela al respecto. Por ello, se partió de la construcción de una representación de esta población y con base en ella se definió la muestra final. Mediante un instrumento de tipo encuesta cerrada, con enfoque cuantitativo, se les consultó a las personas empleadoras respecto al desempeño de las personas graduadas que tienen contratadas, se incluyeron temas de destrezas, conocimientos, habilidades y aptitudes, además de una valoración integral de la calidad de la carrera. 
- Personas expertas en la disciplina: esta población fue abordada mediante un instrumento cualitativo de entrevista a profundidad, que se trabajó con una guía de preguntas y un conversatorio personal en, al menos, dos ocasiones con cada persona. La muestra en este caso se construyó también por los miembros de la comisión, y contempló como principal criterio incluir a personas ingenieras civiles que fueran referentes en las diferentes áreas de la disciplina en el país. Se planteó que al menos se tuviera una persona experta por área, finalmente se logró una muestra de 10 personas. A esta muestra se le consultó sobre el desarrollo general de la carrera en el país y en el mundo, los cambios en la disciplina, el abordaje de la enseñanza de la ingeniería que aplica la Universidad de Costa Rica, entre otros aspectos. Estas personas son expertas en ingeniería civil, que en algún grado y momento de su vida profesional han tenido vinculación con la Universidad, que han recibido el reconocimiento público del Colegio Federado de Ingenieros y de Arquitectos de Costa Rica por los logros en su vida profesional y que en su mayoría están pensionados.

- Personas investigadoras en la disciplina: corresponde a profesionales en ingeniería que se dedican a la academia, y se encuentran en centros de investigación. Se contemplaron representantes nacionales e internacionales. Se elaboró una entrevista que incluyó preguntas abiertas y cerradas. La entrevista se envió primeramente vía correo electrónico y posteriormente se contactó, personalmente, vía Skype con cada seleccionado. Este instrumento responde a un enfoque metodológico mixto, que abarca aspectos cualitativos y cuantitativos para obtener la mayor cantidad de información posible. De una muestra inicial de 10 personas dedicadas a la investigación, se logró respuesta de 6 . No se conoce el número total de individuos de la población, ya que no se llevan registros de la cantidad de profesionales en ingeniería civil que están en centros de investigación activos en el mundo. A ellos se les consultó respecto a los temas de vanguardia que se investigan en la disciplina; además, se les preguntó acerca de las expectativas de cómo va a evolucionar la profesión en los próximos 10 años. Adicionalmente, se les consultó acerca de técnicas de investigación y temáticas que desarrollan.

Específicamente, para el caso de los cuestionarios, fueron montados usando la plataforma gratuita de Google Forms, y se enviaron varios recordatorios para llenar los 
cuestionarios durante los meses que se mantuvieron activos. A continuación, se muestran la cantidad de respuestas obtenidas, para las diferentes poblaciones encuestadas, en el proceso de actualización del plan de estudio de la Escuela de Ingeniería Civil (EIC) de la Universidad de Costa Rica:

Tabla 1

Número y porcentaje de personas consultadas por población de estudio, EIC - UCR

\begin{tabular}{llll}
\hline Población & $\begin{array}{l}\text { Cantidad de personas a las } \\
\text { que se envió la encuesta } \\
\text { satisfactoriamente }\end{array}$ & $\begin{array}{l}\text { Cantidad de personas } \\
\text { que respondieron }\end{array}$ & Equivalente porcentual \\
\hline Estudiantes & 278 & 135 & $49 \%$ \\
Graduados & 1506 & 267 & $18 \%$ \\
Empleadores & 366 & 60 & $16 \%$ \\
Docentes & 74 & 62 & $84 \%$ \\
\hline
\end{tabular}

Fuente: Elaboración propia a partir de los datos recopilados durante las consultas (2017)

Este proceso de consulta se nutrió, además, de una investigación documental que contempló literatura de procesos epistemológicos en ingeniería en otras universidades del mundo, y el estudio de qué se enseña en otras universidades referentes en el ámbito disciplinar. En este último punto, se contempló una muestra de 10 universidades alrededor del mundo.

En todo este proceso se trabajó con triangulación de la información obtenida de las diferentes fuentes. La triangulación metodológica, según comenta Vasilachis (1992, p.65) al citar a otros actores, es la combinación de metodologías para estudiar un mismo objeto con el fin de superar los sesgos propios de cada metodología, y de combinar varias técnicas que permitan el mejor entendimiento de la realidad, donde lo clave radica en lograr la integración de la información. Es por ello que para la presente investigación se buscó una operacionalización múltiple de herramientas que permitieran, a partir de diferentes aristas, entender la realidad del objeto de estudio de la ingeniería civil. Según argumenta Arias (2000, p. 06), los investigadores pueden utilizar la triangulación metodológica cuando "se usan dos o más métodos de investigación [...] la triangulación dentro de métodos es la combinación de dos o más recolecciones de datos, con similares aproximaciones en el mismo estudio para medir una misma variable". 


\section{Resultados y su análisis}

Uno de los primeros resultados que refleja esta investigación es que la epistemología de la ingeniería es un área del conocimiento que integra ciencias básicas, ciencias de la ingeniería, ciencias aplicadas y tecnologías para satisfacer las necesidades sociales, siguiendo teorías y métodos de cada una de ellas. Esto le agrega complejidad al análisis, pues plantea la necesidad de diferenciar la ingeniería de otras ramas del conocimiento.

A partir de la recopilación de información primaria y secundaria, una de las tareas que se realizó, como parte de los resultados, fue el definir dimensiones de la ingeniería, y definir conceptos como qué se entiende por ingeniería, ingeniería civil y sus especialidades, esto con el fin de homologar esa definición de objeto de estudio, que es el primer paso de la construcción epistemológica que se busca con esta investigación. Seguidamente, se exponen los resultados obtenidos:

\subsection{Dimensiones de la Ingeniería}

Al construir las dimensiones de la ingeniería, el abordaje cualitativo fue clave para la recopilación de opiniones diversas. Gracias a que estos métodos:

...dependen fundamentalmente de la observación de los actores en su propio terreno y de la interacción con ellos en su lenguaje y con sus mismos términos...constituye un fenómeno empírico, socialmente localizado, definido positivamente por su propia historia y no negativamente por la carencia de cifras. (Vasilachis, 1992, p. 59)

Con base en esa información primaria, brindada por las personas entrevistadas expertas, investigadoras y docentes, se pudo validar que, para la visión de Ingeniería Civil de la Universidad de Costa Rica, se concuerda con lo que establece Figuereido (2008, p.1), quien plantea una visión muy interesante de la Ingeniería, pues no minimiza la dimensión social que está en su base, sino que, más bien, la retoma como una de sus principales dimensiones, postura que no había sido clara anteriormente para la Escuela (autoridades y su personal docente). Así, este autor propone una epistemología de la ingeniería que incluye 4 dimensiones y que se estarían adoptando como válidas para este proceso:

- Ciencias sociales: como principal diferencia con respecto a las personas tecnólogas, las ingenieras deben abordar una dimensión social en sus proyectos (Figuereido, 2008, p.3), con el fin de reconocer la naturaleza eminentemente social del mundo en 
el que actúan. La creación de valor social y económico, así como la satisfacción de las necesidades sociales son los fines últimos de la Ingeniería.

- Ciencias básicas: se extrae la lógica y la rigurosidad de estas disciplinas para generar conocimiento por medio del análisis y la experimentación. Quien se dedica a la ingeniería debe tener una sólida formación en ciencias básicas para crear nuevos artefactos a partir de métodos heurísticos, como se ha venido mencionando.

- Diseño: también se ha mencionado la importancia de esta dimensión para la ingeniería, diseñar requiere de un proceso previo de configuración mental en la búsqueda de una solución en cualquier campo. Es una conceptualización y planeación de lo que aún no existe, es la integración de requisitos técnicos, sociales y económicos, necesidades biológicas, ergonomía con efectos psicológicos y materiales, forma, color, volumen y espacio, todo ello pensado e interrelacionado con el medio ambiente que rodea a la humanidad.

- Realización práctica: la persona profesional en ingeniería realiza cambios en el mundo, hace frente a los retos y considera las complejidades existentes por medio de flexibilidad y perseverancia. Esto retoma la naturaleza teleológica de la Ingeniería, mencionada anteriormente, así como un lugar fundamental para el pensamiento de la ética.

Estas dimensiones deben entenderse como necesarias en un mundo real, en el que el acercamiento sistémico y transdisciplinario es fundamental para responder a una sociedad cambiante bajo condiciones cada vez más exigentes. Esto ha hecho evolucionar el accionar en la ingeniería, que cada vez más requieren de interactuar con otras profesiones para resolver los problemas a los que se enfrentan. Si diseñar es lo que prioritariamente los distingue de otras áreas, hay que considerar que, cada vez más, diseñar es una tarea interdisciplinaria, compleja, dinámica e intrincada.

La ingeniería civil sería entonces un tipo particular de ingeniería, con fundamentos epistemológicos comunes a ese ámbito mayor, para la resolución de un tipo específico de necesidades sociales, como se verá a continuación. Pero con particularidades específicas para cada una de sus ramas del saber. 


\subsection{Definiciones de Ingeniería y de Ingeniería Civil}

Tal como lo hace Vasilachis (1992, p.17) en su análisis para la sociología, la ingeniería también parte de la investigación científica que intenta responder a las mismas interrogantes: ¿desde qué?, ¿con qué? o ¿con quién?, ¿cómo?, ¿cuándo y dónde?, ¿para qué? y ¿qué? Estas interrogantes guían todos los procesos epistemológicos, por ende, también los de la ingeniería; también guían el accionar de la ingeniería civil, incluso hasta el diseño mismo de las obras.

Como se indicó, las consultas realizadas a actores clave permitieron la construcción de definiciones propias. Estas se derivaban de un amplio proceso de consulta en el seno de la Escuela de Ingeniería Civil y fueron aprobadas en su Asamblea de Escuela. Ambos conceptos (ingeniería e ingeniería civil) deben entenderse en el marco de la discusión epistemológica presentada anteriormente. A continuación se presentan parte de las definiciones que fueron aprobadas para estos conceptos.

\subsubsection{Ingeniería}

Para el contexto de esta investigación, la ingeniería es la disciplina que estudia las necesidades de desarrollo humano sostenible con el propósito de dar soluciones prácticas para la vida humana, procura el uso eficiente de los recursos disponibles para la transformación óptima del ambiente. Se basa en conocimientos científicos y tecnológicos rigurosos de manera contextualizada, en la ética, el sentido común y la creatividad con miras al planteamiento y la resolución de problemas de diferente grado de complejidad.

En este contexto, desarrollo humano sostenible abarca la planeación en el uso de los recursos de manera que se satisfagan las necesidades humanas del presente sin comprometer los recursos para las necesidades futuras. La transformación óptima del ambiente es aquella que busca ser sostenible y generar beneficios económicos y sociales. Se contempla entre los recursos disponibles todos aquellos necesarios para el desarrollo de los proyectos: humanos, naturales, económicos, entre otros.

\subsubsection{Ingeniería Civil}

De igual manera, para el contexto de esta investigación, la ingeniería civil es la rama de la ingeniería que se aboca al diseño y desarrollo de infraestructura (de orden no militar) de manera sostenible, y que considera el ambiente, los materiales para la construcción, los 
sistemas operativos y los aspectos de política pública relacionados. Lo anterior involucra actividades de planificación, diseño, construcción, deconstrucción, inspección, auscultación, preservación, mantenimiento y restauración, tanto de lo construido como del terreno sobre el cual se construye.

Asimismo, la ingeniería civil busca el bien común de la ciudadanía, propicia el desarrollo sostenible en armonía con el ambiente, la conciliación en contextos sociales particulares y la gestión del riesgo involucrado.

\section{3 Áreas y campos de acción de la Ingeniería Civil}

La Ingeniería Civil puede dividirse en áreas de especialización según el tipo de problema en infraestructura y servicios que se aborden. Por medio de un proceso participativo, en la Escuela de Ingeniería Civil de la Universidad de Costa Rica, se establecieron 6 áreas o campos de acción principales, que pueden ser diferentes en otros ámbitos educativos. A continuación, se presentan las definiciones y alcances que se adoptaron para cada una:

- Ingeniería estructural: se encarga de conceptualizar, diseñar, analizar y evaluar los elementos y sistemas estructurales de cualquier obra civil con el propósito de garantizar su funcionalidad, la seguridad para la vida, así como otros aspectos económicos y estéticos.

- Ingeniería de construcción: se encarga de la planeación, la administración y la materialización de obras civiles, para lo cual hace uso de técnicas de construcción y deconstrucción adecuadas para cumplir con las especificaciones del proyecto. Se consideran el proceso y las técnicas constructivas, la prevención de riesgos laborales, los costos y presupuestos involucrados, los equipos de construcción, maquinarias, tasaciones o evaluaciones de proyectos y peritajes, así como la selección y dirección del personal.

- Ingeniería de recursos hidráulicos: se encarga de la planificación, diseño y ejecución de obras relacionadas con el agua para el aprovechamiento, protección y uso del recurso.

- Ingeniería ambiental: se encarga de identificar, conceptualizar, analizar y solucionar problemas que resultan de la interacción entre las actividades que desarrolla el ser 
humano y el ambiente que las circunscribe, esto con el fin de garantizar condiciones sanitarias y ambientales adecuadas para la vida en sociedad.

- Ingeniería de transportes: se encarga de la planificación, diseño, operación, mantenimiento, rehabilitación, desempeño y evaluación de sistemas integrados de transporte, incluidos los pavimentos y materiales, con especial énfasis en aspectos económicos y de política pública.

- Ingeniería geotécnica: se encarga del estudio de las propiedades mecánicas e hidráulicas de los suelos y las rocas para la conceptualización, diseño y construcción de obras de ingeniería, para lo cual se apoya en la mecánica de suelos, la mecánica de rocas, la geología y la ingeniería geológica.

A la luz de estas definiciones queda clara la complejidad de la disciplina en análisis y la diversidad del objeto de estudio que se requiere abarcar. Esto conlleva a pensar que la investigación asociada a la creación de conocimiento en el campo y a la definición de la epistemología en la ingeniería civil requieren de un abordaje profundo y, posteriormente, pasa por una reflexión a lo interno de la unidad académica, para ser complementado con la información primaria recopilada.

\subsection{Los cambios en los métodos y herramientas que usa la ingeniería civil}

De las entrevistas realizadas a personas expertas en las diferentes ramas de la ingeniería civil se concluye que uno de los mayores cambios (y también uno de los mayores retos) para la disciplina es el avance tecnológico en el uso de herramientas. Parte de la habilidad para ejercer la ingeniería radica precisamente en saber utilizar eficientemente esas herramientas para la resolución de problemas. En los últimos 23 años (periodo que tiene el plan de estudios actual de estar vigente) la cantidad de recursos informáticos que vienen a apoyar el quehacer diario de la ingeniería ha aumentado radicalmente. Seis de las diez personas expertas entrevistadas concordaron en que este cambio implica un proceso de reajuste de qué se aprende en ingeniería y cómo se hace. Sin embargo, cabe destacar la postura de uno de ellos al resaltar que es importante no olvidar las bases y conocimientos conceptuales: "en esa carrera por estar a la punta en la tecnología, no se puede olvidar la importancia de tener claridad en los conceptos que hay detrás y en entender lo que está sucediendo en la realidad para simplificarlo y resolverlo". 
Otro cambio importante, que se ha dado en la disciplina y que repercute de forma negativa en la influencia que tienen o mantienen las personas ingenieras en la toma de decisiones en el país, es la falta de interés en la participación política a nivel nacional. Esto tiene consecuencias en el nivel de desarrollo de la infraestructura y en la planificación de nuestras ciudades. A pesar de que el área construida se duplicó en la Gran Área Metropolitana entre los años 80 y 2010, y la densidad poblacional pasó de 68.3 a 75.4 habitantes/hectárea urbana en ese período (Estado de la Nación, 2015, p.275), la inversión en infraestructura no creció de la misma manera, y en parte, según las consultas realizadas, ese rezago se puede atribuir a la falta de dinamismo de los profesionales en ingeniería civil en los ámbitos públicos del país. Esta aseveración indicada por varias de las personas consultadas fue comentada en los talleres con docentes, quienes validaron la afirmación con cautela, lo que llevó a la conclusión de la importancia de valorar dicha falta de dinamismo asociada a la forma cómo se le incentiva al estudiantado la participación en espacios públicos desde las aulas. La participación puede ser una de las herramientas más poderosas para gestionar cambios.

\subsection{Novedades que se perfilan para la disciplina y su relación con otras disciplinas}

De los resultados de las entrevistas a profundidad con personas expertas de la ingeniería civil, se evidenció que cada vez más, el trabajo que se realiza en esta rama del conocimiento requiere de un enfoque interdisciplinario o multidisciplinario, pues los proyectos que se realizan son más complejos y requieren de diferentes perspectivas para afrontarlos exitosamente. Esto plantea la necesidad de reforzar la necesidad de aprender a trabajar los proyectos con otras disciplinas, ya sea con otras ramas de la ingeniería o con disciplinas diferentes como la biología, la sociología, la economía, entre otras. Esta dimensión emergente de la investigación (trabajo multidisciplinario) conlleva a evaluar la necesidad de que esa relación con otras disciplinas esté inmersa también en la formación del estudiantado, y también en la creación de conocimiento en la disciplina.

Más que áreas emergentes en la disciplina, lo que se requiere contemplar a mayor profundidad son temas emergentes, tales como el análisis de riesgos y la vulnerabilidad de las estructuras. Según los resultados de las entrevistas a personas expertas, uno de los mayores retos actuales para la ingeniería civil es el adaptar las obras viejas a los cambios 
del entorno (natural y social) que las rodea. De acuerdo con los resultados de las entrevistas a personas investigadoras, algunas de las nuevas áreas que se perfilan con fuerza están relacionadas con la restauración, adaptación y rehabilitación de estructuras, ya que cada vez más los sitios están ya construidos, intervenidos, y se requiere realizar cambios para mejorar sus usos. Esto plantea retos para la disciplina, que se ha centrado durante muchos años casi exclusivamente en obras nuevas.

Al parecer, según se refleja de la triangulación de información primaria, entre las entrevistas y las encuestas a personas empleadoras, uno de los principales problemas es que las personas graduadas en ingeniería tienen dificultades para visualizar los proyectos en el contexto en el que se deben desarrollar. Más aún cuando este contexto debe responder a cambios abruptos en el entorno social o en los recursos disponibles en un corto tiempo.

Según opinan tanto quienes se dedican a la investigación como las personas expertas entrevistadas, la sostenibilidad de los proyectos también es un área que ha tenido un auge importante en los últimos 10 años y se perfila como una de las que seguirá creciendo en importancia. Esta es una de las áreas que requiere mayor trabajo multidisciplinario, lo que plantea también un reto de integración en la universidad a la hora de llevarlo a las aulas y de aplicarlo en la investigación.

Como parte de la necesidad actual de fomentar proyectos de ingeniería más sostenibles, la eficiencia energética y las alternativas de energías limpias son temas que también presentan un auge en la actualidad. Si bien estos temas están más relacionados al quehacer de otras áreas disciplinares, como la ingeniería eléctrica, es necesario que la ingeniería civil promueva un uso sostenible de la energía en las edificaciones y en la infraestructura en general, tanto en su fase constructiva como durante su ocupación y operación. Así lo hicieron ver tres de las personas investigadoras entrevistadas que describieron este tema como de franco crecimiento en los últimos 5 años y, por lo tanto, una prioridad a inculcar durante la formación de profesionales en ingeniería.

\subsection{Actualidad en la investigación de la disciplina}

Según los resultados obtenidos, algunas de las áreas donde más se está realizando investigación asociada a la ingeniería civil son:

- Gestión y control de residuos, sólidos y líquidos.

- Riesgo de amenazas naturales contra obras civiles. 
- Mitigación y adaptación de infraestructura al cambio climático.

- Modelación avanzada para el análisis de inundaciones urbanas y no urbanas.

- Modelado y mejoramiento de materiales de construcción.

- Aplicación de criterios de sostenibilidad a la construcción.

- Minería de residuos.

Puede constatarse que la investigación en el ámbito de la ingeniería civil continúa abordando problemas que han sido recurrentes en la historia de la disciplina, aunque usando tecnologías y métodos novedosos, y considerando elementos del contexto propios de la actualidad como lo son el cambio climático y sus efectos, o la tendencia actual de incorporar la variable de sostenibilidad en los proyectos.

Otro resultado significativo de la investigación es que, de la totalidad de las personas empleadoras que respondieron la encuesta, ninguna señaló dedicarse a la investigación. Las personas investigadoras y expertas entrevistadas apuntaron en la misma dirección, señalando que en el sector privado, incluso a nivel de la academia, prácticamente no se realiza investigación, y la escasa que se lleva a cabo tiene poca repercusión en la sociedad y en la generación de conocimiento local. Paradójicamente, las personas empleadoras que respondieron la encuesta sí indicaron que para el desempeño profesional de la ingeniería la investigación es importante (36\%) o más o menos importante (48\%).

De los y las docentes que respondieron la encuesta, sobre la relevancia de la habilidad para la investigación que deben tener quienes sales de las aulas a ejercer la disciplina, 46\% respondió que es muy relevante, y $35 \%$ que es algo relevante. Para estar en la academia estos porcentajes pueden considerarse bajos, pues desde allí es donde debe impulsarse esa construcción de conocimiento. A la pregunta de si realizaban algún tipo de investigación, $64 \%$ respondieron afirmativamente. Al describir su labor como investigador, 54\% del personal docente respondió que recibe el apoyo o facilidades que requieren, $72 \%$ indicó que logra involucrar al estudiantado en esas investigaciones, $79 \%$ afirmó que los resultados de lo que investiga ayuda a mejorar los cursos que imparte y $82 \%$ opinó que la investigación aporta al desarrollo de la disciplina.

También cabe destacar que, según las personas expertas e investigadoras entrevistadas, es en la Universidad de Costa Rica y en el Instituto Tecnológico de Costa Rica donde se está llevando a cabo la mayor parte de la investigación del país en la disciplina. Le 
sigue el aporte de otros entes públicos y no gubernamentales, como el Instituto Costarricense de Electricidad y el Colegio Federado de Ingenieros y de Arquitectos. También indicaron que los recursos (principalmente económicos) para apoyar la investigación son escasos y los fondos externos se han reducido en los últimos años, pero esto no ha frenado, aunque sí desacelerado, el desarrollo de las investigaciones.

\subsection{La relación entre los atributos de egreso y la construcción de nuevo conocimiento en la disciplina}

Para que quien es profesional en ingeniería pueda contribuir a la construcción de nuevo conocimiento en el área requiere tener una serie de habilidades que le propicien el crecimiento profesional, aunado a la capacidad de sintetizar esos nuevos conocimientos y transmitirlos a los demás. En esta línea, cabe plantear la siguiente interrogante: ¿el ingenio de la persona ingeniera se posee per se, se tiene desde antes de iniciar su formación, es innato o más bien puede configurarse (es decir educarse, emerger producto de la influencia del proceso educativo al que se le somete, de la experiencia, la práctica, la reflexión, el estudio)? Parte de las dinámicas desarrolladas en los talleres participativos con docentes planteó esta dicotomía, y se llegó a la conclusión de que, desde el punto de vista académico, debería pensarse que dicho ingenio puede y debe desarrollarse en el estudiantado de ingeniería, sin menoscabar los posibles talentos que la persona posea y que le facilitarían durante el proceso de desarrollo de su ingenio.

De los atributos de egreso marcados por la Canadian Engineering Accreditation Board como básicos, y que han sido adoptados por la Escuela, algunos son trascendentales para cumplir lo dicho anteriormente, en especial, los de habilidades de investigación, planificación conceptual, expresión oral y escrita, habilidades de trabajo individual y en equipo, entre otras.

De los cuestionarios realizados (abordaje cuantitativo), otro resultado relevante es la opinión de los diferentes actores respecto a una serie de atributos que debe tener toda persona graduada de la Carrera. Se les solicitó su percepción acerca del nivel de desarrollo de esos atributos en ellos mismos (para estudiantes o personas graduadas) o en sus subalternos y subalternas (en caso de personas docentes y empleadoras), al realizar labores propias de la ingeniería. 
Las Figuras 1 y 2 se presentan los resultados principales de las encuestas realizadas por un medio electrónico. Esto impidió aclarar posibles dudas de los y las participantes y dificultó homologar lo que cada uno de ellos entendía para las diferentes preguntas. Esto pudo generar algún sesgo en la respuesta.

Figura 1

Valoración porcentual de la percepción del desarrollo de los atributos de egreso producto de la formación recibida según población encuestada, EIC-UCR parte 1
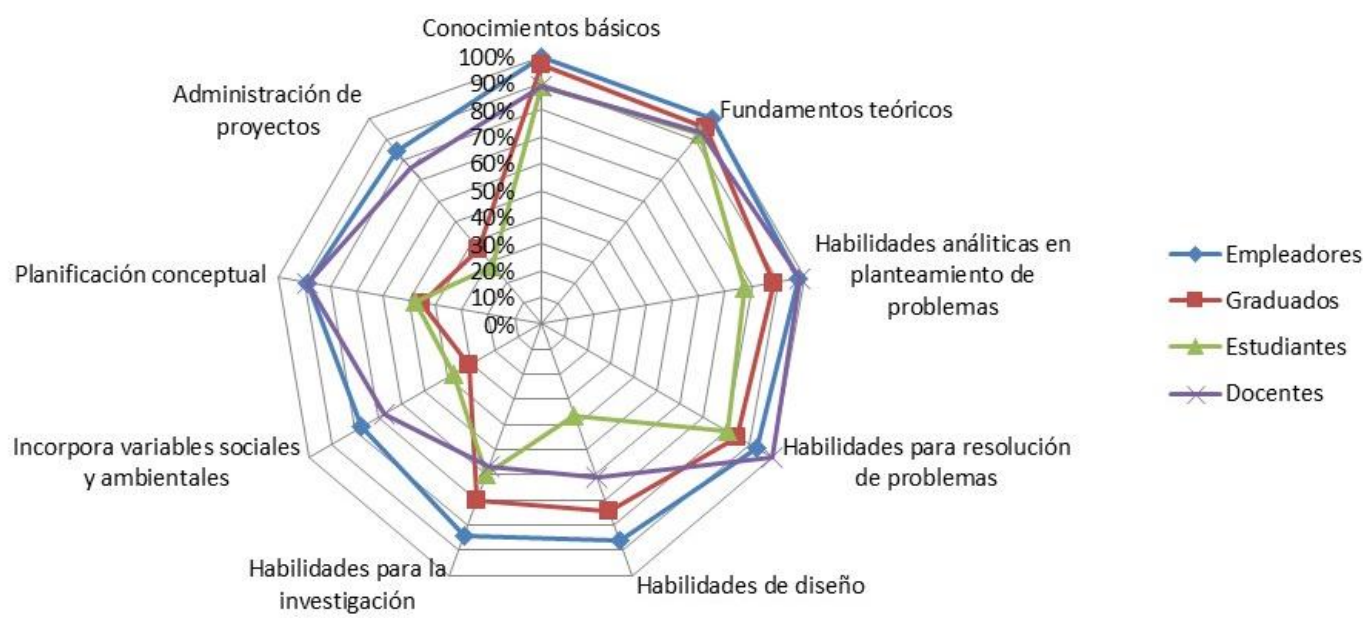

investigación

Fuente: Elaboración propia con base en los resultados de las consultas a poblaciones clave (2017) 
Figura 2

Valoración porcentual de la percepción del desarrollo de los atributos de egreso producto de la formación recibida según población encuestada, EIC-UCR parte 2

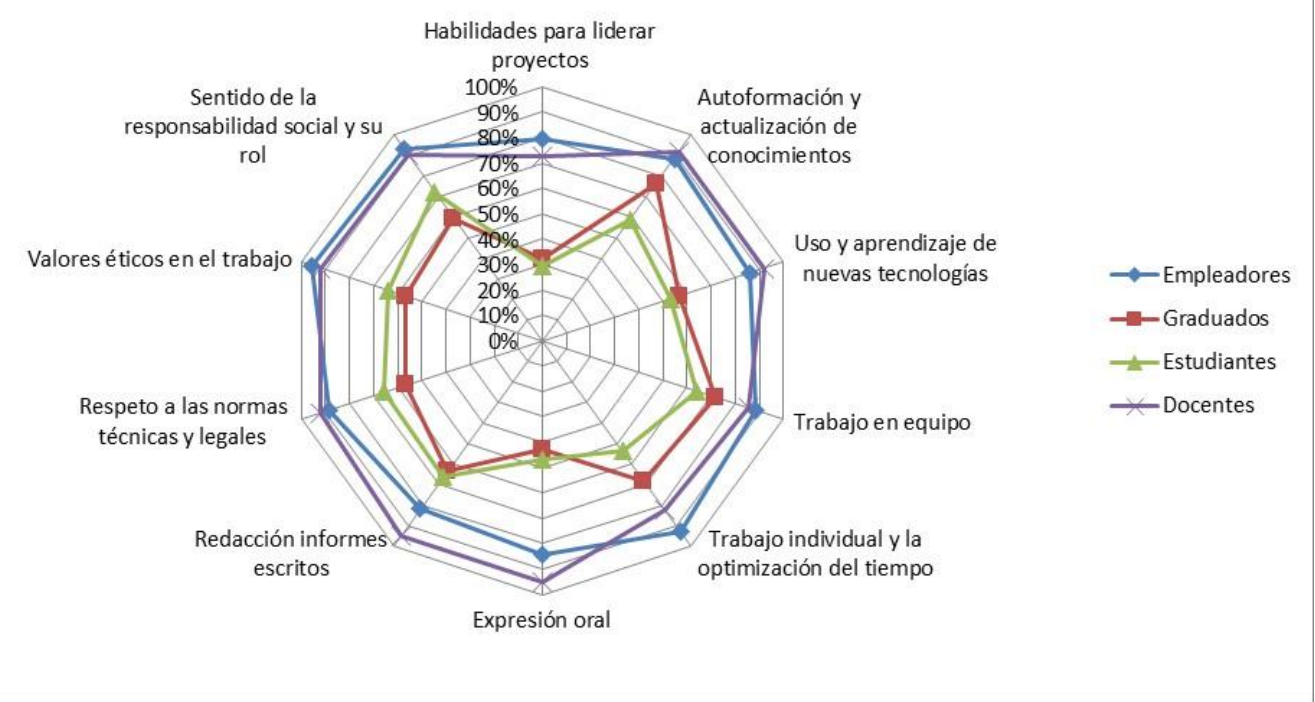

Fuente: Elaboración propia con base en los resultados de las consultas a poblaciones clave (2017)

Los datos de los gráficos anteriores buscan reflejar que tanto cada población considera que la carrera ayuda a formar en dichos atributos. Importa resaltar de estos resultados, cómo las poblaciones que se están evaluando a sí mismas (personas graduadas y estudiantes) suelen ser más críticas. De la información recibida que las poblaciones externas (empleadores y docentes) se tienen valoraciones más positivas. También es muy interesante ver cómo, en general, las habilidades de investigación fueron calificadas con una nota intermedia, pero la habilidad para planificación conceptual y la de liderar proyectos sí son calificadas con puntuación muy baja, en especial, por las poblaciones de estudiantes y personas graduadas. Esto da luces respecto a que atributos deben reforzarse en la formación en ingeniería civil. Estos resultados fueron posteriormente discutidos en los talleres con docentes para ampliar su interpretación, influyendo en la definición de una epistemología de la ingeniería.

\section{Consideraciones finales}

Para entender el trasfondo epistemológico de una disciplina se requiere de un abordaje teórico de base, que sea enriquecido por información de fuentes primarias, para esa construcción del objeto de estudio. La investigación es el pilar que nutre la creación de 
conocimiento en una disciplina. Para la ingeniería civil, la investigación nace y se hace en las universidades, donde se debe generar el cambio en la disciplina. En el país, en general, existen muchas otras universidades que ofrecen la carrera, pero pocas realizan una investigación significativa que enriquezca el desarrollo de la profesión.

Los resultados de la investigación arrojan como conclusión que la disciplina, a pesar de que es muy estable en el tiempo (cambia poco su objeto de estudio), está inmersa en un mundo tecnológico al que debe responder, y en el que las herramientas computacionales juegan un papel crucial en la forma cómo se aprende e investiga en esta y otras disciplinas. Los resultados a los que se ha llegado con esta investigación tienen implicaciones respecto a cómo se propicia una investigación más crítica y centrada en las necesidades de la sociedad costarricense desde la perspectiva de la ingeniería civil. A pesar de que se tienen grandes aportes con labores de este tipo, realizadas por centros como el Laboratorio Nacional de Materiales y Modelos Estructurales (LANAMME) de la Universidad de Costa Rica y otros, se requiere profundizar aún más. En suma, se requiere trabajar en los principios éticos y precautorios en materia ambiental para que quienes ejercen la ingeniería civil los apliquen en su quehacer diario, esto debe enfatizarse desde la Universidad.

Una futura ampliación de la presente investigación podría profundizar más en la aplicación de estos resultados a la evaluación por atributos. Las tendencias mundiales en las ingenierías están enfocándose en ese camino, y así como lo citaron muchas de las personas investigadoras y expertas, parte de las oportunidades de mejora dadas en el desempeño de las personas ingenieras civiles radica en formarlas mejor en competencias blandas como el liderazgo, la escritura y la oralidad. El propio atributo de investigación es clave a la hora de seguir construyendo la epistemología en la ingeniería civil. Así, si desde etapas tempranas de la formación en ingeniería civil no se les enseña a investigar, es poco probable que la futura persona profesional esté buscando como ampliar sus conocimientos y propiciar la construcción de nuevo conocimiento para la resolución de sus retos diarios.

\section{Referencias}

Arias, María Mercedes (2000). La triangulación metodológica: sus principios, alcances y limitaciones. https://www.uv.mx/mie/files/2012/10/Triangulacionmetodologica.pdf

Bulleit, William. (2012). What Makes an Engineering Education an Engineering Education? In Structures Congress March 29-31, 1143-1151. DOI: 10.1061/9780784412367.102 
Coronado, Guillermo. (2002). Perspectivas en ciencia, tecnología y ética. Cartago, Costa Rica: Editorial Tecnológica de Costa Rica.

Covarrubias, José Manuel. (1998). Tres documentos sobre la formación de ingenieros. Ingenierias, $1(1), \quad 5-9 . \quad$ Recuperado de http://ingenierias.uanl.mx/1/pdf/1_Jose_Cobarruvias_Tres_documentos.pdf

Dias, Priyan. (2013). The Engineer's Identity Crisis: Homo Faber or Homo Sapiens. In Diane Michelfelder, Natasha McCarthy and David Goldberg (eds.), Philosophy and Engineering: Reflections on Practice, Principles and Process (pp. 139-150). DOI: 10.1007/978-94-007-7762-0_11

Figueiredo, Antonio D. de. (2008). Toward an Epistemology of Engineering. 2008 Workshop on Philosophy and Engineering, The Royal Academy of Engineering. London, November 10-12, Recuperado de https://ssrn.com/abstract=1314224

Galland, Gustavo Adolfo. (2011). Reflexiones Epistemologias: En torno a la cuestión de la ciencia y la tecnología. XXXIX Congresso Brasileiro de Educação Em Engenharia. Recuperado de http://www.abenge.org.br/cobenge/arquivos/8/sessoestec/art1854.pdf

Herrera Jiménez, Rodolfo. (2016). Tecnología / Ingeniería: Un Estudio Epistemológico. Cartago, Costa Rica: Editorial Tecnológica de Costa Rica.

Koen, Billy Vaughn. (2003). Discussion of the Method: Conducting the engineer's approach to problem solving. Nueva York, Estados Unidos: Oxford University Press.

Poser, Hans. (1998). On structural differences between science and engineering. PHIL \& TECH, 4(2), 81-93. DOI: https://doi.org/10.5840/techne1998426

Prince Cruzat, Sergio y Llach Valdivieso, Carolina (2006). El estatus epistemológico de la ingeniería y su importancia para el diseño Curricular. Chile: Universidad de Valparaíso. Recuperado de https://www.yumpu.com/es/document/view/13743782/el-estatusepistemologico-de-la-ingenieria-y-su-eici

Programa de Estado de la Nación. (2015). Capítulo 6: Ordenamiento territorial: Implicaciones para el Desarrollo Humano. Recuperado de http://www.estadonacion.or.cr/21/assets/en-21-cap-6-en-2015.pdf

Tapella, Esteban. (2007). El mapeo de Actores Claves (Documento de trabajo del proyecto Efectos de la biodiversidad funcional sobre procesos ecosistémicos, servicios ecosistémicos y sustentabilidad en las Américas: un abordaje interdisciplinario). Universidad Nacional de Córdoba. Recuperado de https://planificacionsocialunsj.files.wordpress.com/2011/09/quc3a9-es-el-mapeo-deactores-tapella1.pdf

Vasilachis de Gialdino, Irene. (1992). Métodos Cualitativos I los problemas teóricosepistemológicos. Buenos Aires, Argentina: Centro Editor de América Latina S.A. 
Vasilachis de Gialdino, Irene. (2009). Los fundamentos ontológicos y epistemológicos de la investigación cualitativa. Forum Qualitative Sozialforschung, 10(2), Recuperado de: http://www.qualitative-research.net/index.php/fqs/article/view/1299/2778

Velásquez, Luis José y D’ Armas, Mayra. (2015). El ingeniero con consciencia social. Una posibilidad para el desarrollo sostenible. Universidad, Ciencia y Tecnología, 19(74). Recuperado de http://www.scielo.org.ve/scielo.php?script=sci arttext\&pid=S1316$\underline{48212015000100003}$ 